\title{
Long time clinical outcomes of limus-eluting stent versus paclitaxel-eluting stent in patients undergoing percutaneous coronary artery intervention: A meta-analysis of randomized controlled clinical trials
}

\author{
Lei Gao*, Xin $\mathrm{Hu}^{*}$, Yuntian Hou, Qiao Xue
}

Institute of Geriatric Cardiology, Chinese PLA General Hospital, Beijing, China

\begin{abstract}
Background: The meta-analysis was aimed to compare the long time (> 2 year) clinical outcomes of limus-based stents (LBS) and paclitaxel-eluting stents (PES). LBS and PES are two kinds of most common coronary artery stents in clinics.

Methods: Electronic data bases of PubMed, Cochrane, and EMBASE were searched. We included randomized controlled clinical trials (RCT) comparing LBS and PES with long time clinical outcomes. Methodological quality of eligible trials was assessed using "risk of bias" tool. The efficacy endpoints included target lesion revascularization (TLR), target vessel revascularization (TVR), and stent thrombosis (ST), and the safety endpoints included all cause death, cardiac death, and myocardial infarction (MI). Odds ratios (OR) and 95\% confidence intervals (95\% CI) were calculated for each endpoint.

Results: A total of 23 RCTs and 19,319 participants were included and analyzed in this meta-analysis. All trials were of an acceptable quality. At 2 years, LBS showed reduced risk of revascularization and $S T$ (TLR: OR $=0.59,95 \%$ CI 0.44-0.78; TVR: OR $=0.63,95 \%$ CI 0.55-0.71; ST: OR $=0.55,95 \%$ CI 0.41-0.73) and a lower rate of $M I(O R=0.67,95 \%$ CI 0.57-0.79). Subgroup analysis showed that both everolimus-eluting stents and sirolimus-eluting stents had better clinical outcomes compared with PES. The comparisons of 3, 4 and 5 year follow-up results revealed that the clinical outcomes of PES were non-inferior to those of LBS but LBS was associated with reduced risk of MI and ST at 3 years.
\end{abstract}

Conclusions: LBS is associated with better clinical outcomes at 2 years. Both LBS and PES showed similar efficacy and safety at long time period. (Cardiol J 2014; 21, 3: 211-219)

Key words: target vessel revascularization, limus-based stents, paclitaxel-eluting stents, percutaneous coronary intervention

\footnotetext{
Address for correspondence: Dr Lei Gao, Institute of Geriatric Cardiology, General Hospital of People's Liberation Army, No. 28 Fuxing Road, Hai dian District, Beijing 100853, P.R. China, tel: 0086-10-55499339, fax: 0086-10-55499339, e-mail: nkgaolei2010@126.com

*These authors contributed equally to this work.

Received: 07.06.2013 Accepted: 13.07.2013
} 


\section{Introduction}

The application of drug-eluting stents (DES) is a landmark event of percutaneous coronary intervention (PCI). Compared with bare metal stents (BMS), DES is associated with a significantly reduced risk of restenosis and target-vessel revascularization (TVR) in patients undergoing PCI $[1,2]$. Although the first generation DES showed a perfect solution at the beginning, meta-analysis and randomized controlled trials showed that the rate of very late ( $>1$ year) stent thrombosis (ST) was increased with first generation DES [3, 4].

Paclitaxel-eluting stents (PES) and sirolimus-eluting stents (SES), are recognized as first generation DES, because they were first introduced to the market and approved. Thereafter, a number of DES was developed and introduced to the market, such as everolimus-eluting stents (EES) and zotarolimus-eluting stents (ZES). Generally, limus-based stents (LBS) and PES are the two kinds of DES most commonly used in clinical. These two kinds of coronary stents inhibit in stent restenosis by different pharmacological mechanisms. Paclitaxel is an antineoplastic drug interfering cell microtubule function [5, 6]. Paclitaxel alters the dynamic equilibrium between microtubules and alpha- and beta-tubulin by favoring the formation of abnormally stable microtubules [7]. In vivo studies showed that paclitaxel could inhibit neointimal proliferation at a very low blood concentration [8]. On the other hand, limus can also inhibit neointimal proliferation. Sirolimus is a potent immunosuppressant agent, which induces expression of the cyclin-dependent kinase inhibitor, p27 kip1, blocks the cell cycle at the G1 to S phase [9]. Everolimus can also exert anti-proliferative effect by inhibiting cell proliferation and inducing cell cycle arrest in the late G1 stage [10].

Given different pharmacological mechanisms, the clinical outcomes of PES and LBS may alter over time, especially in a long time period. However, there has been no meta-analysis comparing DES with respect to pharmacological mechanisms. A lot of meta-analyses have compared the 1 year clinical outcomes of SES, EES, and PES [11-13]. Thus, we performed this meta-analysis to compare the long time (2-5 years) efficacy and safety of the LBS and PES in patients with PCI.

\section{Methods}

\section{Searching strategy}

Electronic databases of PubMed, Cochrane, and EMBASE were searched for eligible rando- mized controlled clinical trials (RCTs). There was no limitation on languages and the last search was performed on 18 September 2012. The following key words were used "sirolimus", "everolimus", "zotarolimus", "paclitaxel”, and "stent”. The references lists of relative articles were also searched for additional trials.

\section{Inclusion and exclusion criteria}

Trials met the following inclusion criteria: 1) RCTs; 2) comparison of LBS and PES in patients with PCI; 3) clinical outcomes of 2 years follow-up or more. The trials met the following exclusion criteria: 1) subgroup results of RCTs; 2) non-randomized; 3) without clinical outcomes.

\section{Data collection}

Two reviewers independently collected data with a standard data-collection form and reached consensus on each item. The following data were collected: first author, publishing time, stent type, time of follow-up, number of participants, age, male, number of patients with diabetes, number of patients analyzable, target lesion revascularization (TLR), TVR, stent thrombosis (ST), all cause death, cardiac death, and myocardial infarction (MI).

\section{Assessment of risk of bias}

The methodological quality of eligible trials was assessed using the "risk of bias" tool, recommended by the Cochrane Collaboration. Adequate sequence generation, allocation concealment, blinding, incomplete outcome data addressed, and free of selective reporting were assessed. Each of the 5 items was grades as "yes", "no", or "unknown", representing "low", "high", or "uncertain of" risk.

\section{Statistical analysis}

The efficacy and safety endpoints of LBS and PES in this meta-analysis were TLR, TVR, ST, all cause death, cardiac death, and MI at 2, 3, 4, and 5 years of follow-up. Odds ratios (OR) and 95\% confidence intervals (CI) were calculated as summary statistics. Pooled OR was calculated using the Mantel-Hænzel fixed effects model or DerSimonian-Laird random effects model. In the absence of heterogeneity across trials, fixed effects model was used, otherwise, we chose random effects model. The $\chi^{2}$ test was performed to test the heterogeneity across included trials, and a $p<0.10$ showed a significant heterogeneity. For publication bias, a graphic funnel plot was used to detect the existence of publication bias. Subgroup analyses (SES vs. PES, EES vs. PES, ZES vs. PES) were 


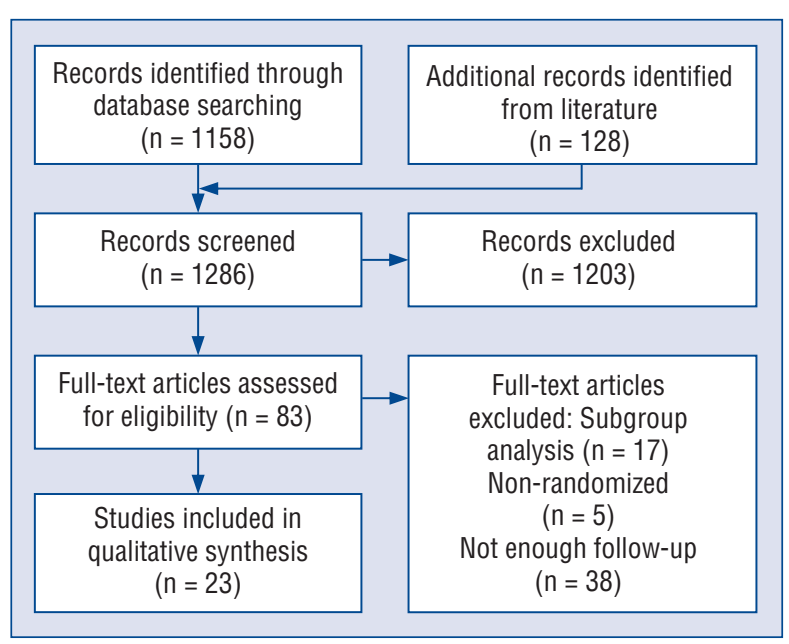

Figure 1. Flow chart.

performed to explore the difference of specific type of stents.

\section{Results}

\section{Trial flow}

A number of 1286 records form databases and references lists were screened. After screening of titles and abstracts, 82 records were extracted for further evaluation. In the end, 23 eligible trials [14-36] were included and analyzed. The detailed process of trial flow is shown in Figure 1 (flow chart).

\section{Characteristics of eligible trials and risk of bias}

A number of 23 trials and 19319 patients were included in our meta-analysis. There were 3 trials [14-16] with 5-year clinical outcomes, and 2 trials $[17,18]$ with 4 year-clinical outcomes, 7 trials [19-25] with 3 year-outcomes, and 11 trials [26-36] with 2 year-clinical results. SES was compared in 14 trials and EES was compared in 8 trials. Main characteristics of included trials are shown in Table 1. Methodological quality of eligible trials was acceptable (shown in Table 2). Most trials addressed the method for sequence generation and allocation concealment.

\section{Clinical outcomes at 2 years}

The 2-year follow-up results were available in 11 trials with 13251 participants. As for revascularization and ST, LBS was superior to PES at 2 years. There were 375 patients with LBS and 406 patients with PES received TLR at 2 years of follow-up (OR $=0.59$, 95\% CI 0.44-0.78; Fig. 2). LBS was also associated with lower risk of TVR $(\mathrm{OR}=0.63,95 \% \mathrm{CI} 0.55-0.71)$ and ST $(\mathrm{OR}=0.55$, 95\% CI 0.41-0.73). In subgroup analysis, both SES and EES showed a lower rate TLR and TVR compared with PES, but only EES was associated with a reduced risk of ST $(\mathrm{OR}=0.33,95 \%$ CI $0.20-0.52)$. In terms of safety, LBS was associated with a lower risk of $\mathrm{MI}(\mathrm{OR}=0.67,95 \%$ CI $0.57-0.79)$, but LBS and PES showed equal results of all-cause death and cardiac death. Subgroup analysis revealed that EES and ZES reduced the risk of MI but not SES (Table 3 ).

In conclusion, compared with PES, LBS showed a lower risk of revascularization and ST at 2-year follow-up and significantly reduced the incidence of MI. As for subgroup analysis, SES and EES but not ZES were superior to PES in TLR, TVR and ST.

\section{Clinical outcomes at 3 years}

A total of 7 trials and 3718 patients were analyzed for 3-year clinical outcomes. LBS and PES had equal clinical outcomes at 3-year follow-up. One hundred and twenty-eight and 112 patients received TLR at 3 years in the groups of LBS and PES, respectively. Patients with LBS had similar rate of TLR $(\mathrm{OR}=0.80,95 \% \mathrm{CI} 0.46-1.41$; Fig. 3$)$, TVR (OR $=0.83,95 \%$ CI $0.66-1.04)$, and ST $(\mathrm{OR}=0.78,95 \%$ CI 0.50-1.22) compared to those with PES. In subgroup analyses, SES, EES, and ZES all showed similar efficacy with PES in the comparison of TLR, TVR, and ST. LBS and PES did not differ significantly in the rate of all cause death $(\mathrm{OR}=0.83,95 \% \mathrm{CI} 0.61-1.14)$, but LBS was associated with lower risk of $\mathrm{MI}(\mathrm{OR}=0.53,95 \% \mathrm{CI}$ 0.37-0.75). Subgroup analysis showed that only ZES showed a reduced risk of $\mathrm{MI}(\mathrm{OR}=0.43,95 \% \mathrm{CI}$ 0.24-0.79). LBS and PES did not differ significantly in most 3-year clinical outcomes, but the rate of MI was lower with LBS, especially for ZES (Table 3).

\section{Clinical outcomes at 4 years}

Two trials reported 4-year follow-up clinical outcomes of 662 patients. In summary, LBS showed lower risk of TLR $(\mathrm{OR}=0.53,95 \% \mathrm{CI}$ $0.31-0.92)$ but similar risk of ST (OR $=1.35,95 \%$ CI 0.46-3.98) compared to PES. Both LBS and PES showed similar safety, regarding the rate of cardiac death $(\mathrm{OR}=0.58,95 \% \mathrm{CI} 0.20-1.63)$ and $\mathrm{MI}$ $(\mathrm{OR}=0.69,95 \% \mathrm{CI} 0.26-1.84)$. In the presence of only 2 trials, subgroup analysis was not performed. LBS was associated a lower rate of TLR compared with PES at 4-year follow-up, but they did not differ significantly in other clinical outcomes (Table 3 ). 
Table 1. Characteristics of eligible trials.

\begin{tabular}{|c|c|c|c|c|c|c|c|}
\hline Author & Year & $\begin{array}{l}\text { Follow up } \\
\text { [years] }\end{array}$ & Comparison & Patients & Age [years] & Male & Diabetes \\
\hline Sun-Joo Jang & 2012 & 2 & $\begin{array}{c}\text { Zotarolimus } \\
\text { Sirolimus } \\
\text { Paclitaxel }\end{array}$ & $\begin{array}{l}883 \\
878 \\
884\end{array}$ & $\begin{array}{l}61.7+9.3 \\
61.9 \pm 9.6 \\
62.0 \pm 9.6\end{array}$ & $\begin{array}{c}66.40 \% \\
67.30 \% \\
65.85\end{array}$ & $\begin{array}{l}30.40 \% \\
28.10 \% \\
27.70 \%\end{array}$ \\
\hline Lamin King & 2012 & 5 & $\begin{array}{l}\text { Sirolimus } \\
\text { Paclitaxel }\end{array}$ & $\begin{array}{l}225 \\
225\end{array}$ & $\begin{array}{l}66.8 \pm 10.5 \\
66.6 \pm 10.2\end{array}$ & $\begin{array}{l}75 \% \\
79 \%\end{array}$ & $\begin{array}{l}68 \% \\
74 \%\end{array}$ \\
\hline Ki-Woon Kang & 2012 & 5 & $\begin{array}{l}\text { Sirolimus } \\
\text { Paclitaxel }\end{array}$ & $\begin{array}{l}105 \\
121\end{array}$ & $\begin{array}{l}60 \pm 9 \\
62 \pm 8\end{array}$ & $\begin{array}{c}34.30 \% \\
43.8 \%\end{array}$ & $\begin{array}{l}34.30 \% \\
34.70 \%\end{array}$ \\
\hline Lorenz Räber & 2011 & 5 & $\begin{array}{l}\text { Sirolimus } \\
\text { Paclitaxel }\end{array}$ & $\begin{array}{l}503 \\
509\end{array}$ & $\begin{array}{l}62 \pm 11 \\
62 \pm 12\end{array}$ & $\begin{array}{l}76 \% \\
78 \%\end{array}$ & $\begin{array}{l}22 \% \\
18 \%\end{array}$ \\
\hline Gregg W. Stone & 2011 & 2 & $\begin{array}{c}\text { Everolimus } \\
\text { Paclitaxel }\end{array}$ & $\begin{array}{l}2458 \\
1229\end{array}$ & $\begin{array}{l}63.3 \pm 10.5 \\
63.3 \pm 10.2\end{array}$ & $\begin{array}{l}67.70 \% \\
67.80 \%\end{array}$ & $\begin{array}{l}32.00 \% \\
32.50 \%\end{array}$ \\
\hline Pieter C. Smits & 2011 & 2 & $\begin{array}{c}\text { Everolimus } \\
\text { Paclitaxel }\end{array}$ & $\begin{array}{l}897 \\
903\end{array}$ & $\begin{array}{l}62.9 \\
63.6\end{array}$ & $\begin{array}{l}69 \% \\
72 \%\end{array}$ & $\begin{array}{l}17 \% \\
19 \%\end{array}$ \\
\hline Seung-Whan Lee & 2011 & 4 & $\begin{array}{l}\text { Sirolimus } \\
\text { Paclitaxel }\end{array}$ & $\begin{array}{l}200 \\
200\end{array}$ & $\begin{array}{l}61.1 \pm 8.9 \\
60.7 \pm 8.8\end{array}$ & $\begin{array}{l}61.00 \% \\
55.00 \%\end{array}$ & $\begin{array}{l}100 \% \\
100 \%\end{array}$ \\
\hline Carlo Briguori & 2011 & 3 & $\begin{array}{c}\text { Sirolimus } \\
\text { Everolimus } \\
\text { Paclitaxel }\end{array}$ & $\begin{array}{l}76 \\
75 \\
75\end{array}$ & $\begin{array}{c}64 \pm 8 \\
65 \pm 8 \\
64 \pm 10\end{array}$ & $\begin{array}{l}57 \% \\
56 \% \\
59 \%\end{array}$ & $\begin{array}{l}100 \% \\
100 \% \\
100 \%\end{array}$ \\
\hline Robert J. Applegate & 2011 & 3 & $\begin{array}{c}\text { Everolimus } \\
\text { Paclitaxel }\end{array}$ & $\begin{array}{l}669 \\
332\end{array}$ & $\begin{array}{l}63.2 \pm 10.5 \\
62.8 \pm 10.2\end{array}$ & $\begin{array}{l}70.10 \% \\
65.70 \%\end{array}$ & $\begin{array}{l}29.60 \% \\
27.90 \%\end{array}$ \\
\hline Martin B. Leon & 2010 & 3 & $\begin{array}{c}\text { Zotarolimus } \\
\text { Paclitaxel }\end{array}$ & $\begin{array}{l}773 \\
775\end{array}$ & $\begin{array}{l}64 \pm 11 \\
64 \pm 11\end{array}$ & $\begin{array}{l}66.90 \% \\
68.50 \%\end{array}$ & $\begin{array}{l}31.20 \% \\
30.50 \%\end{array}$ \\
\hline Scot Garg & 2011 & 4 & $\begin{array}{c}\text { Everolimus } \\
\text { Paclitaxel }\end{array}$ & $\begin{array}{l}195 \\
67\end{array}$ & $\begin{array}{c}62 \pm 10 \\
62 \pm 9\end{array}$ & $\begin{array}{l}71 \% \\
79 \%\end{array}$ & $\begin{array}{l}23 \% \\
24 \%\end{array}$ \\
\hline Soon Jun Hong & 2010 & 3 & $\begin{array}{l}\text { Sirolimus } \\
\text { Paclitaxel }\end{array}$ & $\begin{array}{l}85 \\
84\end{array}$ & $\begin{array}{l}65.9 \pm 8.0 \\
64.5 \pm 8.9\end{array}$ & $\begin{array}{l}71.80 \% \\
76.20 \%\end{array}$ & $\begin{array}{l}100 \% \\
100 \%\end{array}$ \\
\hline Davran Çiçek & 2010 & 2 & $\begin{array}{l}\text { Sirolimus } \\
\text { Paclitaxel }\end{array}$ & $\begin{array}{l}103 \\
101\end{array}$ & $\begin{array}{l}57 \pm 10.9 \\
58 \pm 10.2\end{array}$ & $\begin{array}{l}\text { NA } \\
\text { NA }\end{array}$ & $\begin{array}{l}39 \% \\
36 \%\end{array}$ \\
\hline Martin B. Leon & 2009 & 2 & $\begin{array}{c}\text { Zotarolimus } \\
\text { Paclitaxel }\end{array}$ & $\begin{array}{l}742 \\
739\end{array}$ & $\begin{array}{l}63.5 \pm 11.1 \\
63.6 \pm 11.0\end{array}$ & $\begin{array}{l}66.90 \% \\
68.50 \%\end{array}$ & $\begin{array}{l}31.20 \% \\
30.50 \%\end{array}$ \\
\hline Scot Garg & 2009 & 3 & $\begin{array}{c}\text { Everolimus } \\
\text { Paclitaxel }\end{array}$ & $\begin{array}{l}195 \\
69\end{array}$ & $\begin{array}{c}62 \pm 10 \\
62 \pm 9\end{array}$ & $\begin{array}{l}71 \% \\
79 \%\end{array}$ & $\begin{array}{l}23 \% \\
24 \%\end{array}$ \\
\hline Bimmer E. Claessen & 2009 & 2 & $\begin{array}{c}\text { Everolimus } \\
\text { Paclitaxel }\end{array}$ & $\begin{array}{l}211 \\
73\end{array}$ & $\begin{array}{c}62 \pm 10 \\
62 \pm 9\end{array}$ & $\begin{array}{l}71 \% \\
79 \%\end{array}$ & $\begin{array}{l}23 \% \\
24 \%\end{array}$ \\
\hline Hyun-Sook Kim & 2011 & 3 & $\begin{array}{l}\text { Sirolimus } \\
\text { Paclitaxel }\end{array}$ & $\begin{array}{l}154 \\
154\end{array}$ & $\begin{array}{l}60 \pm 11 \\
60 \pm 12\end{array}$ & $\begin{array}{l}76.00 \% \\
76.60 \%\end{array}$ & $\begin{array}{l}22.10 \% \\
28.60 \%\end{array}$ \\
\hline Emilio Di Lorenzo & 2009 & 2 & $\begin{array}{l}\text { Sirolimus } \\
\text { Paclitaxel }\end{array}$ & $\begin{array}{l}90 \\
90\end{array}$ & $\begin{array}{l}62 \pm 15 \\
63 \pm 15\end{array}$ & $\begin{array}{l}68.90 \% \\
71.10 \%\end{array}$ & $\begin{array}{l}23.30 \% \\
27.80 \%\end{array}$ \\
\hline Julinda Mehilli & 2009 & 2 & $\begin{array}{l}\text { Sirolimus } \\
\text { Paclitaxel }\end{array}$ & $\begin{array}{l}305 \\
302\end{array}$ & $\begin{array}{l}69.3 \pm 9.34 \\
68.8 \pm 10.1\end{array}$ & $\begin{array}{l}80 \% \\
75 \%\end{array}$ & $\begin{array}{l}28 \% \\
30 \%\end{array}$ \\
\hline Seung-Whan Lee & 2009 & 2 & $\begin{array}{l}\text { Sirolimus } \\
\text { Paclitaxel }\end{array}$ & $\begin{array}{l}200 \\
200\end{array}$ & $\begin{array}{l}61.1 \pm 8.9 \\
60.7 \pm 8.8\end{array}$ & $\begin{array}{l}61.00 \% \\
55.00 \%\end{array}$ & $\begin{array}{l}100 \% \\
100 \%\end{array}$ \\
\hline Gregg W. Stone & 2009 & 2 & $\begin{array}{c}\text { Everolimus } \\
\text { Paclitaxel }\end{array}$ & $\begin{array}{l}642 \\
309\end{array}$ & $\begin{array}{l}63.2 \pm 10.5 \\
62.8 \pm 10.2\end{array}$ & $\begin{array}{l}70.10 \% \\
65.70 \%\end{array}$ & $\begin{array}{l}29.60 \% \\
27.90 \%\end{array}$ \\
\hline Michael Billinger & 2008 & 2 & $\begin{array}{l}\text { Sirolimus } \\
\text { Paclitaxel }\end{array}$ & $\begin{array}{l}503 \\
509\end{array}$ & $\begin{array}{l}\text { NA } \\
\text { NA }\end{array}$ & $\begin{array}{l}75.90 \% \\
78.4 \%\end{array}$ & $\begin{array}{l}100 \% \\
100 \%\end{array}$ \\
\hline Alexandre Berger & 2007 & 3 & $\begin{array}{l}\text { Sirolimus } \\
\text { Paclitaxel }\end{array}$ & $\begin{array}{l}100 \\
102\end{array}$ & $\begin{array}{l}65 \pm 10 \\
63+10\end{array}$ & $\begin{array}{l}77 \% \\
83 \%\end{array}$ & $\begin{array}{l}\text { NA } \\
\text { NA }\end{array}$ \\
\hline
\end{tabular}

Patients show the number of subjects available for meta-analysis in each trial; age is presented as mean and standard deviate; NA — not available

\section{Clinical outcomes at 5 years}

Three trials reported 5-year follow-up results of 1688 patients. And 3 trials all compared SES and
PES, thus, subgroup analysis was not performed. There was no statistical difference between the clinical outcomes of LBS and PES at 5 years. They did not 
Table 2. Risk of bias assessment.

\begin{tabular}{|c|c|c|c|c|c|c|}
\hline Author & Year & $\begin{array}{l}\text { Adequate } \\
\text { sequence } \\
\text { generation }\end{array}$ & $\begin{array}{l}\text { Allocation } \\
\text { concealment }\end{array}$ & Blinding & $\begin{array}{l}\text { Incomplete } \\
\text { outcome data } \\
\text { addressed }\end{array}$ & $\begin{array}{l}\text { Free of selective } \\
\text { reporting }\end{array}$ \\
\hline Sun-Joo Jang & 2012 & $\mathrm{Y}$ & $\mathrm{Y}$ & $\mathrm{Y}$ & $Y$ & $\mathrm{Y}$ \\
\hline Lamin King & 2012 & $\mathrm{Y}$ & Y & Y & $\mathrm{Y}$ & Y \\
\hline Ki-Woon Kang & 2012 & $\mathrm{Y}$ & $U$ & $\mathrm{Y}$ & $\mathrm{Y}$ & $\mathrm{Y}$ \\
\hline Lorenz Räber & 2011 & $U$ & $U$ & $U$ & Y & $\mathrm{Y}$ \\
\hline Gregg W. Stone & 2011 & $\mathrm{Y}$ & $\mathrm{Y}$ & $\mathrm{Y}$ & $\mathrm{Y}$ & Y \\
\hline Pieter C. Smits & 2011 & $\mathrm{Y}$ & $\mathrm{Y}$ & $\mathrm{Y}$ & Y & $\mathrm{Y}$ \\
\hline Seung-Whan Lee & 2011 & $\mathrm{Y}$ & $\mathrm{Y}$ & $\mathrm{Y}$ & $\mathrm{Y}$ & $\mathrm{Y}$ \\
\hline Carlo Briguori & 2011 & $\mathrm{Y}$ & $\mathrm{Y}$ & $\mathrm{Y}$ & $\mathrm{Y}$ & $\mathrm{Y}$ \\
\hline Robert J. Applegate & 2011 & Y & $\mathrm{Y}$ & $\mathrm{Y}$ & $\mathrm{Y}$ & $\mathrm{Y}$ \\
\hline Martin B. Leon & 2010 & $\mathrm{Y}$ & $\mathrm{Y}$ & $\mathrm{Y}$ & $\mathrm{Y}$ & $\mathrm{Y}$ \\
\hline Scot Garg & 2011 & $\mathrm{Y}$ & $\mathrm{Y}$ & Y & Y & $\mathrm{Y}$ \\
\hline Soon Jun Hong & 2010 & $\mathrm{Y}$ & $U$ & $\mathrm{Y}$ & $\mathrm{Y}$ & $\mathrm{Y}$ \\
\hline Davran Çiçek & 2010 & $U$ & U & $U$ & $Y$ & $\mathrm{Y}$ \\
\hline Martin B. Leon & 2009 & $\mathrm{Y}$ & $\mathrm{Y}$ & $\mathrm{Y}$ & $\mathrm{Y}$ & $\mathrm{Y}$ \\
\hline Scot Garg & 2009 & $U$ & $\mathrm{Y}$ & $\mathrm{Y}$ & $\mathrm{Y}$ & $\mathrm{Y}$ \\
\hline Bimmer E. Claessen & 2009 & $\mathrm{Y}$ & $\mathrm{Y}$ & $\mathrm{Y}$ & $\mathrm{Y}$ & $\mathrm{Y}$ \\
\hline Hyun-Sook Kim & 2011 & $\mathrm{Y}$ & $\mathrm{Y}$ & $\mathrm{Y}$ & Y & $\mathrm{Y}$ \\
\hline Emilio Di Lorenzo & 2009 & $\mathrm{Y}$ & $\mathrm{Y}$ & $\mathrm{Y}$ & $\mathrm{Y}$ & $\mathrm{Y}$ \\
\hline Julinda Mehilli & 2009 & $\mathrm{Y}$ & $\mathrm{Y}$ & $\mathrm{Y}$ & Y & $\mathrm{Y}$ \\
\hline Seung-Whan Lee & 2009 & Y & $\mathrm{Y}$ & $\mathrm{Y}$ & $\mathrm{Y}$ & $\mathrm{Y}$ \\
\hline Gregg W. Stone & 2009 & $\mathrm{Y}$ & $\mathrm{Y}$ & $\mathrm{Y}$ & Y & $\mathrm{Y}$ \\
\hline Michael Billinger & 2008 & $\mathrm{Y}$ & $\mathrm{Y}$ & $\mathrm{Y}$ & $\mathrm{Y}$ & $\mathrm{Y}$ \\
\hline Alexandre Berger & 2007 & $U$ & $U$ & $\mathrm{Y}$ & $Y$ & $Y$ \\
\hline
\end{tabular}

Y for low risk of bias; $U$ for unknown risk of bias

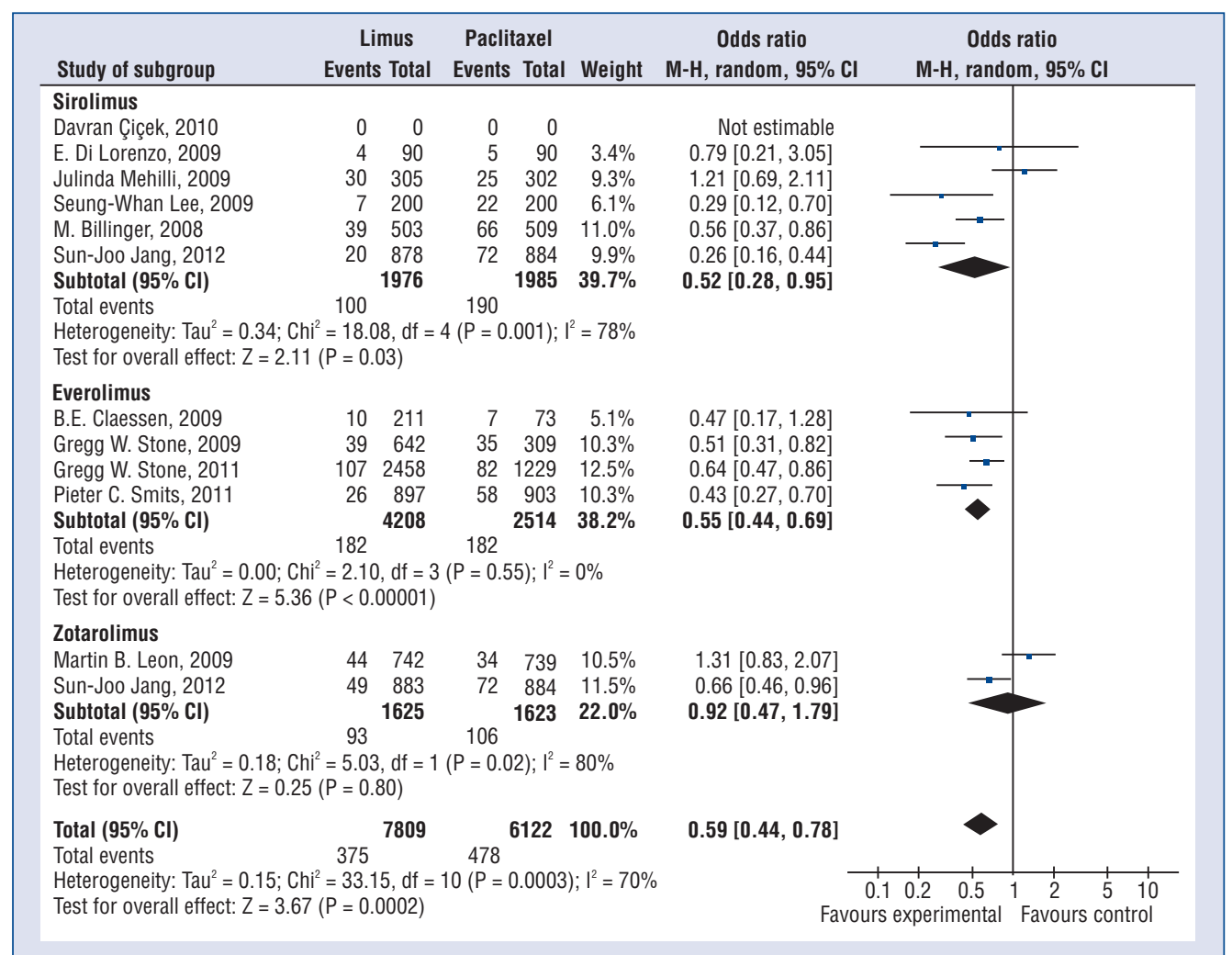

Figure 2. Forest plot for comparison of target lesion revascularization at 2 years. Sizes of data markers are proportional to the weight of each study in the meta-analysis. Horizontal bars indicate $95 \%$ confidence interval. 
Table 3. Meta-analysis results.

\begin{tabular}{|c|c|c|c|c|c|c|c|}
\hline & Subgroup & $\begin{array}{c}\text { Target lesion } \\
\text { revascula- } \\
\text { rization }\end{array}$ & $\begin{array}{c}\text { Target vessel } \\
\text { revascula- } \\
\text { rization }\end{array}$ & $\begin{array}{c}\text { Stent } \\
\text { thrombus }\end{array}$ & All death & Cardiac death & $\begin{array}{l}\text { Myocardial } \\
\text { infarction }\end{array}$ \\
\hline \multirow[t]{4}{*}{2 years } & & $\begin{array}{c}0.59 \\
{[0.44-0.78]}\end{array}$ & $\begin{array}{c}0.63 \\
{[0.55-0.71]}\end{array}$ & $\begin{array}{c}0.55 \\
{[0.41-0.73]}\end{array}$ & $\begin{array}{c}0.90 \\
{[0.73-1.11]}\end{array}$ & $\begin{array}{c}0.92 \\
{[0.68-1.25]}\end{array}$ & $\begin{array}{c}0.67 \\
{[0.57-0.79]}\end{array}$ \\
\hline & SES vs. PES & $\begin{array}{c}0.52 \\
{[0.28-0.95]}\end{array}$ & $\begin{array}{c}0.46 \\
{[0.35-0.60]}\end{array}$ & $\begin{array}{c}0.67 \\
{[0.41-1.10]}\end{array}$ & $\begin{array}{c}0.88 \\
{[0.63-1.23]}\end{array}$ & $\begin{array}{c}0.75 \\
{[0.42-1.33]}\end{array}$ & $\begin{array}{c}0.83 \\
{[0.63-1.09]}\end{array}$ \\
\hline & EES vs. PES & $\begin{array}{c}0.55 \\
{[0.44-0.69]}\end{array}$ & $\begin{array}{c}0.64 \\
{[0.53-0.77]}\end{array}$ & $\begin{array}{c}0.33 \\
{[0.20-0.52]}\end{array}$ & $\begin{array}{c}0.85 \\
{[0.63-1.16]}\end{array}$ & $\begin{array}{c}0.96 \\
{[0.62-1.48]}\end{array}$ & $\begin{array}{c}0.55 \\
{[0.43-0.71]}\end{array}$ \\
\hline & ZES vs. PES & $\begin{array}{c}0.92 \\
{[0.47-1.79]}\end{array}$ & $\begin{array}{c}0.81 \\
{[0.63-1.05]}\end{array}$ & $\begin{array}{c}0.95 \\
{[0.52-1.74]}\end{array}$ & $\begin{array}{c}1.10 \\
{[0.67-1.79]}\end{array}$ & $\begin{array}{c}1.12 \\
{[0.57-2.21]}\end{array}$ & $\begin{array}{c}0.68 \\
{[0.49-0.94]}\end{array}$ \\
\hline \multirow[t]{4}{*}{3 years } & & $\begin{array}{c}0.80 \\
{[0.46-1.41]}\end{array}$ & $\begin{array}{c}0.83 \\
{[0.66-1.04]}\end{array}$ & $\begin{array}{c}0.78 \\
{[0.50-1.22]}\end{array}$ & $\begin{array}{c}0.83 \\
{[0.61-1.14]}\end{array}$ & NA & $\begin{array}{c}0.53 \\
{[0.37-0.75]}\end{array}$ \\
\hline & SES vs. PES & $\begin{array}{c}0.66 \\
{[0.12-3.68]}\end{array}$ & $\begin{array}{c}0.72 \\
{[0.41-1.26]}\end{array}$ & $\begin{array}{c}0.83 \\
{[0.36-1.92]}\end{array}$ & $\begin{array}{c}1.05 \\
{[0.59-1.86]}\end{array}$ & NA & $\begin{array}{c}0.48 \\
{[0.21-1.09]}\end{array}$ \\
\hline & EES vs. PES & $\begin{array}{c}0.81 \\
{[0.34-1.92]}\end{array}$ & $\begin{array}{c}0.78 \\
{[0.53-1.14]}\end{array}$ & $\begin{array}{c}0.83 \\
{[0.34-2.05]}\end{array}$ & $\begin{array}{c}0.63 \\
{[0.36-1.09]}\end{array}$ & NA & $\begin{array}{c}0.65 \\
{[0.39-1.09]}\end{array}$ \\
\hline & ZES vs. PES & $\begin{array}{c}1.10 \\
{[0.72-1.68]}\end{array}$ & $\begin{array}{c}0.91 \\
{[0.65-1.27]}\end{array}$ & $\begin{array}{c}0.72 \\
{[0.38-1.39]}\end{array}$ & $\begin{array}{c}0.88 \\
{[0.53-1.46]}\end{array}$ & NA & $\begin{array}{c}0.43 \\
{[0.24-0.79]}\end{array}$ \\
\hline 4 years & & $\begin{array}{c}0.53 \\
{[0.31-0.92]}\end{array}$ & N/A & $\begin{array}{c}1.35 \\
{[0.46-3.98]}\end{array}$ & N/A & $\begin{array}{c}0.58 \\
{[0.20-1.63]}\end{array}$ & $\begin{array}{c}0.69 \\
{[0.26-1.84]}\end{array}$ \\
\hline 5 years & & $\begin{array}{c}0.90 \\
{[0.67-1.21]}\end{array}$ & $\begin{array}{c}0.41 \\
{[0.11-1.53]}\end{array}$ & $\begin{array}{c}0.89 \\
{[0.53-1.50]}\end{array}$ & $\begin{array}{c}0.88 \\
{[0.46-1.67]}\end{array}$ & $\begin{array}{c}1.09 \\
{[0.80-1.47]}\end{array}$ & $\begin{array}{c}1.09 \\
{[0.74-1.61]}\end{array}$ \\
\hline
\end{tabular}

Results are presented as odds ration and 95\% confidence intervals; SES — sirolimus; EES — everolimus; ZES - zotarolimus; PES — paclitaxel; NA - not available

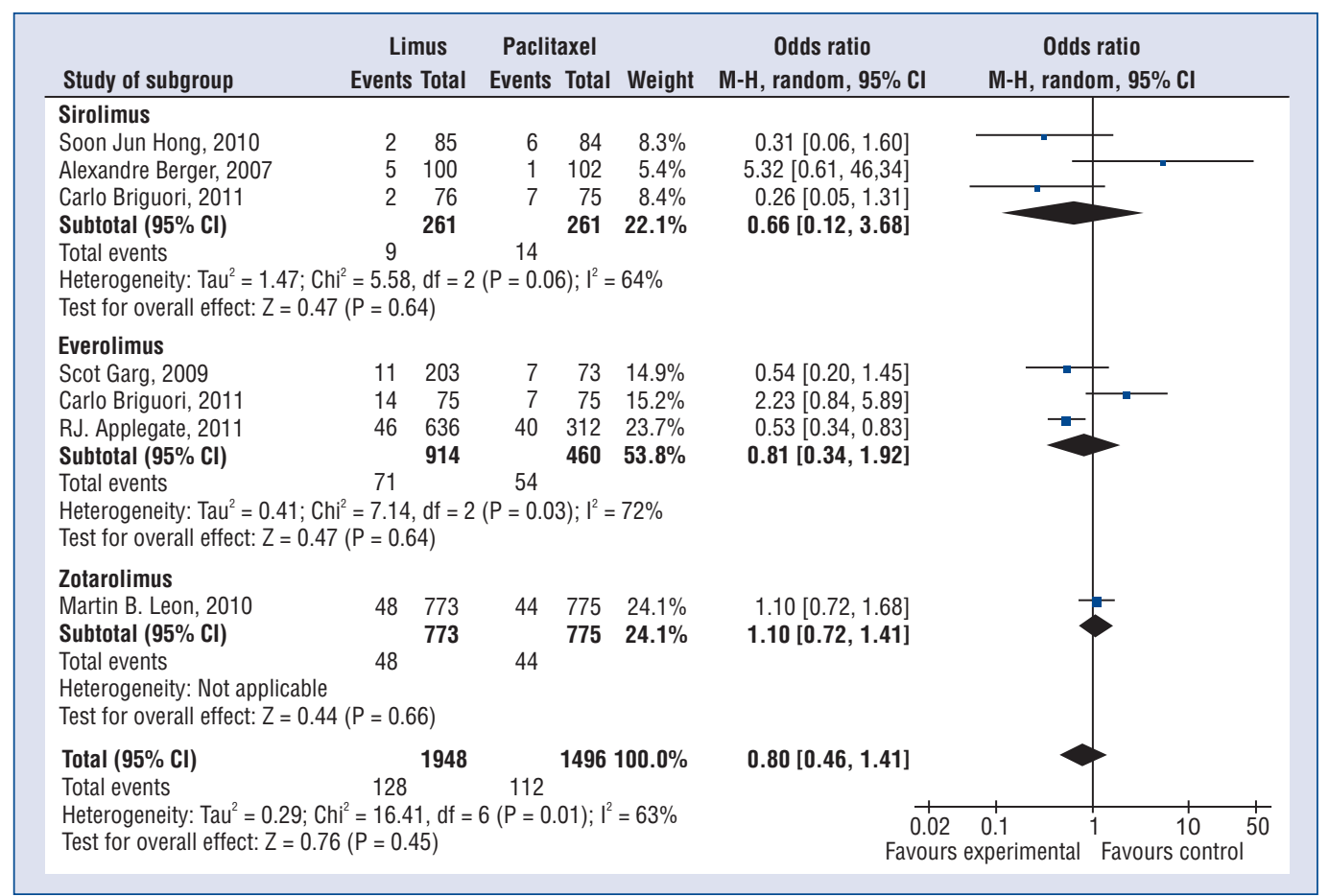

Figure 3. Forest plot for comparison of target lesion revascularization at 3 years. Sizes of data markers are proportional to the weight of each study in the meta-analysis. Horizontal bars indicate $95 \%$ confidence interval.

differ much regarding the rate of TLR $(\mathrm{OR}=0.90$, 95\% CI 0.67-1.21; Fig. 4), TVR (OR $=0.41,95 \%$ CI $0.11-1.53)$, or ST $(\mathrm{OR}=0.89,95 \%$ CI $0.53-1.50)$.
In terms of all cause death $(\mathrm{OR}=1.09,95 \% \mathrm{CI}$ $0.74-1.61)$, cardiac death $(\mathrm{OR}=0.88,95 \% \mathrm{CI}$ 0.46-1.67), and MI (OR = 1.09, 95\% CI 0.80-1.47), 


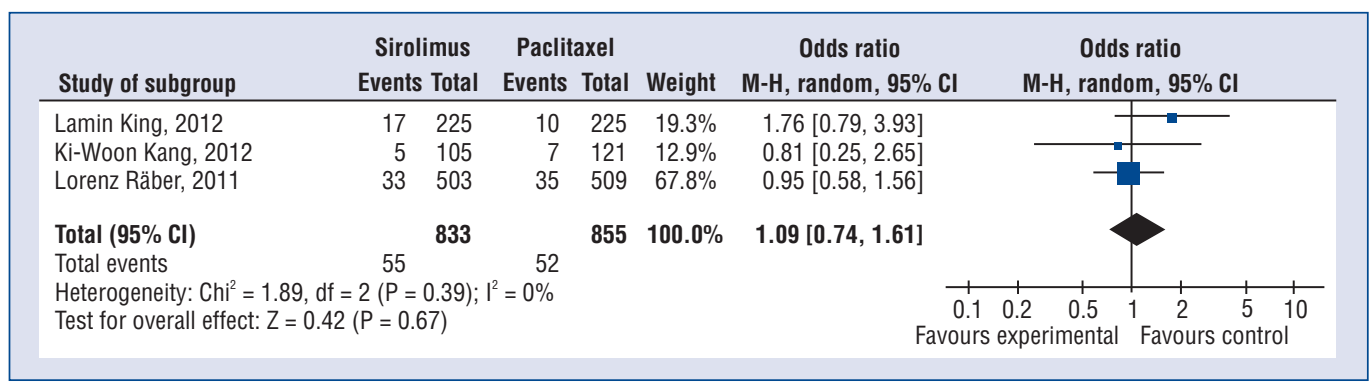

Figure 4. Forest plot for comparison of myocardial infarction at 5 years. Sizes of data markers are proportional to the weight of each study in the meta-analysis. Horizontal bars indicate $95 \%$ confidence interval.

no significant difference was found between LBS and PES (Table 3). In conclusion, there was no significant difference between LBS and PES at 5-year follow-up.

\section{Heterogeneity and publication bias}

Heterogeneity of major comparisons was tested and no severe heterogeneity existed across trials. Funnel plot of each comparison was performed and viewed for symmetry. All of the funnel plots were roughly symmetrical (figures not shown).

\section{Discussion}

This meta-analysis compared long time clinical outcomes of LBS and PES among patients undergoing PCI. We included 23 RCTs and 19319 patients, efficacy and safety of LBS and PES were analyzed at 2, 3, 4, and 5-year follow-up.

DES was a breakthrough technology in the invasive management of coronary artery diseases. LBS and PES are the two most common DES in clinical practice. As mentioned before, the neointimal proliferation pharmacological mechanisms differ significantly between LBS and PES [8-10]. Thus, it is reasonable to hypothesize that LBS and PES would have different clinical outcomes, especially over a long period of time. Pooled results from meta-analyses have showed that LBS and PES had different efficacy and safety at 1 year [11-13], but comparison of long time period was rare. This meta-analysis firstly compared clinical outcomes of DES in a long time period and most importantly, we first compared different types of DES according to pharmacological mechanisms. We provided a new perspective of comparing coronary stents and the long time clinical results would be useful for development of new DES.

According to the comparison of 2- to 5-year clinical outcomes, we could find that clinical outcomes of LBS and PES alter with time and LBS and PES have equally long time efficacy and safety. Previous meta-analyses have showed that LBS was associated with lower efficacy, such as ST and reintervention, compared with PES [11-13]. At 2 years, our meta-analysis results of 11 trials suggested that LBS still had the advantage over PES in the respect of efficacy (TLR, TVR, and ST); additionally, LBS was also associated with a reduced risk of MI. Subgroup analysis suggested that SES and EES significantly reduced the risk of revascularization compared with PES, but ZES was not statistically different from PES in most of the clinical outcomes. Especially EES was also associated with a reduced risk of ST compared with PES. At 3 and 5 years of follow-up, LBS and PES did not differ significantly in most of the clinical outcomes. What is of note is that ZES significantly reduced the risk of $\mathrm{MI}$ in comparison with PES. The 4-year comparison showed some discrepancy and we suspected that the cause was that too few trials were included.

One important conclusion that can be drawn from our meta-analysis is that LBS is non-inferior to PES in terms of very long-time clinical outcomes. This adds to our knowledge about the longstanding clinical outcomes of stents and may change our opinions on the selection of stents. Thus far, only SES and EES were analyzed as LBS. In the comparisons of 5 -year clinical outcomes, only trials comparing SES and PES were analyzed and trials comparing SES, EES and PES were included in the comparison of 3 and 4 years.

We also noticed that although LBS and PES had similar rate of all-cause death and cardiac death, the rate of MI was significantly lower with LBS at 2 and 3 years. This may be explained by the lower rate of ST in the patients with LBS. 


\section{Limitations of the study}

Limitations of this meta-analysis should also be noted before the application of the conclusion. Firstly, aggregate data were collected from each randomized trial and no further subgroup analysis could be performed, such as gender, age, smoking and other risk factors. Although the characteristics of participants of individual study were balanced, it was impossible for us to test whether the baseline characteristics were balanced between the groups. Secondly, trials with 4- and 5-year follow-up were rare and this may cause a discrepancy. The 3 trials analyzed for 5-year clinical outcomes were all designed to compare SES and PES, which also weakened the representativeness of LBS. Thirdly, the meta-analysis was based on randomized trials, not individual patient's data, and we could not assess the confounding factors.

\section{Conclusions}

Based on different pharmacological mechanisms, we systematically compared the long time clinical outcomes of LBS and PES. We found that LBS was associated with better efficacy (TLR, TVR, and ST) and lower rate of MI at 2 years, but comparisons of long time results revealed that both PES and LBS provided similar efficacy and safety. This meta-analysis as the first provides a long time systematical comparison of 2 different stents and it will be useful for future development of new DES.

\section{Conflict of interest: none declared}

\section{References}

1. Moses JW, Leon MB, Popma JJ et al. Sirolimus-eluting stents versus standard stents in patients with stenosis in a native coronary artery. N Engl J Med, 2003; 349: 1315-1323.

2. Stone GW, Ellis SG, Cannon L et al. Comparison of a polymer-based paclitaxel-eluting stent with a bare metal stent in patients with complex coronary artery disease: a randomized controlled trial. JAMA, 2005; 294: 1215-1223.

3. Camenzind E, Steg PG, Wijns W. Stent thrombosis late after implantation of first-generation drug-eluting stents: A cause for concern. Circulation, 2007; 115: 1440-1455; discussion 1455.

4. Stone GW, Moses JW, Ellis SG et al. Safety and efficacy of sirolimus- and paclitaxel-eluting coronary stents. N Engl J Med, 2007; 356: 998-1008.

5. Rowinsky EK, Donehower RC. Paclitaxel (taxol). N Engl J Med, 1995; 332: 1004-1014.

6. Wani MC, Taylor HL, Wall ME, Coggon P, McPhail AT. Plant antitumor agents. VI. The isolation and structure of taxol, a novel antileukemic and antitumor agent from Taxus brevifolia. J Am Chem Soc, 1971; 93: 2325-2327.

7. Schiff PB, Horwitz SB. Taxol stabilizes microtubules in mouse fibroblast cells. Proc Natl Acad Sci USA, 1980; 77: 1561-1565.
8. Sollott SJ, Cheng L, Pauly RR et al. Taxol inhibits neointimal smooth muscle cell accumulation after angioplasty in the rat. J Clin Invest, 1995; 95: 1869-1876.

9. Luo Y, Marx SO, Kiyokawa $\mathrm{H}$ et al. Rapamycin resistance tied to defective regulation of p27Kip1. Mol Cell Biol, 1996; 16: 6744-6751.

10. Farb A, John M, Acampado E et al. Oral everolimus inhibits in-stent neointimal growth. Circulation, 2002; 106: 2379-2384.

11. Cassese S, Piccolo R, Galasso G, De Rosa R, Piscione F. Twelve-month clinical outcomes of everolimus-eluting stent as compared to paclitaxel- and sirolimus-eluting stent in patients undergoing percutaneous coronary interventions. A meta-analysis of randomized clinical trials. Int J Cardiol, 2011; 150: 84-89.

12. Zhang F, Dong L, Qian J, Ge J. Clinical safety and efficacy of everolimus-eluting stents compared to paclitaxel-eluting stents in patients with coronary artery disease. Ann Med, 2011; 43: 75-79.

13. Palmerini T, Kirtane AJ, Serruys PW et al. Stent thrombosis with everolimus-eluting stents: Meta-analysis of comparative randomized controlled trials. Circ Cardiovasc Interv, 2012; 5: 357-364.

14. King L, Byrne RA, Mehilli J et al. Five-year clinical outcomes of a polymer-free sirolimus-eluting stent versus a permanent polymer paclitaxel-eluting stent: Final results of the intracoronary stenting and angiographic restenosis: Test equivalence between two drug-eluting stents (ISAR-TEST) trial. Catheter Cardiovasc Interv, 2012; 81: E23-E28.

15. Kang KW, Ko YG, Shin DH et al. Impact of positive peri-stent vascular remodeling after sirolimus-eluting and paclitaxel-eluting stent implantation on 5-year clinical outcomes: intravascular ultrasound analysis from the Poststent Optimal Stent Expansion Trial multicenter randomized trial. Circ J, 2012; 76: 1102-1108.

16. Raber L, Wohlwend L, Wigger M et al. Five-year clinical and angiographic outcomes of a randomized comparison of sirolimus-eluting and paclitaxel-eluting stents: results of the Sirolimus-Eluting Versus Paclitaxel-Eluting Stents for Coronary Revascularization LATE trial. Circulation, 2011; 123: 2819-2828.

17. Lee SW, Park SW, Kim YH et al. A randomized comparison of sirolimus- versus paclitaxel-eluting stent implantation in patients with diabetes mellitus: 4-year clinical outcomes of DES-DIABETES (drug-eluting stent in patients with DIABETES mellitus) trial. J Am Coll Cardiol Cardiovasc Interv, 2011; 4: 310-316.

18. Garg S, Serruys PW, Miquel-Hebert K. Four-year clinical follow-up of the XIENCE V everolimus-eluting coronary stent system in the treatment of patients with de novo coronary artery lesions: The SPIRIT II trial. Catheter Cardiovasc Interv, 2011; 77: 1012-1017.

19. Briguori C, Airoldi F, Visconti G et al. Novel approaches for preventing or limiting events in diabetic patients (Naples-diabetes) trial: A randomized comparison of 3 drug-eluting stents in diabetic patients. Circ Cardiovasc Interv, 2011; 4: 121-129.

20. Applegate RJ, Yaqub M, Hermiller JB et al. Long-term (three-year) safety and efficacy of everolimus-eluting stents compared to paclitaxel-eluting stents (from the SPIRIT III Trial). Am J Cardiol, 2011; 107: 833-840.

21. Leon MB, Nikolsky E, Cutlip DE et al. Improved late clinical safety with zotarolimus-eluting stents compared with paclitaxel-eluting stents in patients with de novo coronary lesions: 3-year follow-up from the ENDEAVOR IV (Randomized Comparison of Zotarolimus- and Paclitaxel-Eluting Stents in Patients With Coronary Artery Disease) trial. JACC Cardiovasc Interv, 2010; 3: 1043-1050.

22. Hong SJ, Kim MH, Cha KS et al. Comparison of three-year clinical outcomes between sirolimus-versus paclitaxel-eluting stents 
in diabetic patients: prospective randomized multicenter trial. Catheter Cardiovasc Interv, 2010; 76: 924-933.

23. Garg S, Serruys P, Onuma Y et al. 3-year clinical follow-up of the XIENCE V everolimus-eluting coronary stent system in the treatment of patients with de novo coronary artery lesions: the SPIRIT II trial (Clinical Evaluation of the Xience V Everolimus Eluting Coronary Stent System in the Treatment of Patients with de novo Native Coronary Artery Lesions). J Am Coll Cardiol Cardiovasc Interv, 2009; 2: 1190-1198.

24. Kim HS, Lee JH, Lee SW et al. Long-term safety and efficacy of sirolimus- vs. paclitaxel-eluting stent implantation for acute ST-elevation myocardial infarction: 3-year follow-up of the PROSIT trial. Int J Cardiol, 2011; 147: 253-257.

25. Berger A, Stauffer JC, Seydoux C, et al. Three-year follow-up of the first prospective randomized comparison between paclitaxel and sirolimus stents: the TAXi-LATE trial. Catheter Cardiovasc Interv 2007; 70: 163-166.

26. Jang SJ, Park DW, Kim WJ et al. Differential long-term outcomes of zotarolimus-eluting stents compared with sirolimus-eluting and paclitaxel-eluting stents in diabetic and non-diabetic patients: 2-year subgroup analysis of the ZEST randomized trial. Catheter Cardiovasc Interv, 2013; 81: 1106-1114.

27. Stone GW, Rizvi A, Sudhir K et al. Randomized comparison of everolimus- and paclitaxel-eluting stents. 2-year follow-up from the SPIRIT (Clinical Evaluation of the XIENCE V Everolimus Eluting Coronary Stent System) IV trial. J Am Coll Cardiol, 2011; 58: 19-25.

28. Smits PC, Kedhi E, Royaards KJ et al. 2-year follow-up of a randomized controlled trial of everolimus- and paclitaxel-eluting stents for coronary revascularization in daily practice. COMPARE (Comparison of the everolimus eluting XIENCE-V stent with the paclitaxel eluting TAXUS LIBERTE stent in all-comers: a randomized open label trial). J Am Coll Cardiol, 2011; 58: 11-18.

29. Cicek D, Pekdemir H, Kalay N et al. Efficacy of sirolimus-eluting stents compared with paclitaxel-eluting stents in an unselected population with coronary artery disease: 24 -month outcomes of patients in a prospective non-randomized registry in Southern Turkey. Int J Med Sci, 2010; 7: 191-196.

30. Leon MB, Kandzari DE, Eisenstein EL et al. Late safety, efficacy, and cost-effectiveness of a zotarolimus-eluting stent compared with a paclitaxel-eluting stent in patients with de novo coronary lesions: 2-year follow-up from the ENDEAVOR IV trial (Randomized, Controlled Trial of the Medtronic Endeavor Drug [ABT-578] Eluting Coronary Stent System Versus the Taxus Paclitaxel-Eluting Coronary Stent System in De Novo Native Coronary Artery Lesions). J Am Coll Cardiol Cardiovasc Interv, 2009; 2: 1208-1218.

31. Claessen BE, Beijk MA, Legrand V et al. Two-year clinical, angiographic, and intravascular ultrasound follow-up of the XIENCE $\mathrm{V}$ everolimus-eluting stent in the treatment of patients with de novo native coronary artery lesions: The SPIRIT II trial. Circ Cardiovasc Interv, 2009; 2: 339-347.

32. Di Lorenzo E, De Luca G, Sauro R et al. The PASEO (PaclitAxel or Sirolimus-Eluting Stent Versus Bare Metal Stent in Primary Angioplasty) randomized trial. J Am Coll Cardiol Cardiovasc Interv, 2009; 2: 515-523.

33. Mehilli J, Kastrati A, Byrne RA et al. Paclitaxel- versus sirolimus-eluting stents for unprotected left main coronary artery disease. J Am Coll Cardiol, 2009; 53: 1760-1768.

34. Lee SW, Park SW, Kim YH et al. A randomized comparison of sirolimus- versus paclitaxel-eluting stent implantation in patients with diabetes mellitus 2-year clinical outcomes of the DES-DIABETES trial. J Am Coll Cardiol, 2009; 53: 812-813.

35. Stone GW, Midei M, Newman W et al. Randomized comparison of everolimus-eluting and paclitaxel-eluting stents: two-year clinical follow-up from the Clinical Evaluation of the Xience V Everolimus Eluting Coronary Stent System in the Treatment of Patients with de novo Native Coronary Artery Lesions (SPIRIT) III trial. Circulation, 2009; 119: 680-686.

36. Billinger M, Beutler J, Taghetchian KR et al. Two-year clinical outcome after implantation of sirolimus-eluting and paclitaxel-eluting stents in diabetic patients. Eur Heart J, 2008; 29: 718-725. 